\title{
Relevance of selenoprotein transcripts for selenium status in humans
}

\author{
Edyta Reszka • Ewa Jablonska • \\ Jolanta Gromadzinska $\cdot$ Wojciech Wasowicz
}

Received: 5 July 2011/Accepted: 19 August 2011/Published online: 7 September 2011

(C) The Author(s) 2011. This article is published with open access at Springerlink.com

\begin{abstract}
The most commonly used methods for assessing the selenium (Se) status in humans involve analysis of Se concentration, selenoprotein activity, and concentration in the blood and its compartments. Recently, it has been suggested that the expression of selenoprotein mRNA in circulating blood leukocytes could differently reflect Se status, due to prioritization of specific selenoprotein synthesis in response to dietary Se supply. Whereas the Se levels required for optimization of selenoprotein $\mathrm{P}$ level and plasma glutathione peroxidise activity are well known, estimation of Se level that is required for maximal mRNA expression of selenoprotein in humans is the subject of current investigations. Studies on rats suggest that whole blood selenoprotein mRNA level can be used as the relevant molecular biomarker for assessing Se status, and suboptimal Se intake may be sufficient to achieve effective expression. Human studies, however, did not confirm this hypothesis. According to studies on rodents and humans discussed in this review, it appears that suboptimal Se intake may be sufficient to satisfy molecular requirements of Se and it is lower than current recommended dietary intake in humans. The use of selenoprotein transcripts as a molecular biomarker of Se status requires further studies on a large group of healthy individuals with different baseline $\mathrm{Se}$, including data regarding genetic polymorphism of selenoproteins and data regarding potential modifiers of Se metabolism.
\end{abstract}

E. Reszka $\cdot$ E. Jablonska · J. Gromadzinska ·

W. Wasowicz $(\square)$

Department of Toxicology and Carcinogenesis, Nofer Institute of Occupational Medicine, 8 Teresy St., 91-348 Lodz, Poland e-mail: wojciech@imp.lodz.pl
Keywords Selenium - Selenoproteins - Transcripts · Polymorphism

\section{Introduction}

Discovered by Berzelius in 1817 , selenium (Se) belongs to the 16 group (formerly group 6A) in the periodic table, together with oxygen, sulfur, tellurium, and polonium (Nogueira and Rocha 2011). Initially, Se was considered only as a toxic element, but for several decades it has been known as an essential trace element associated with significant health benefits in humans and mammals (Schwarz and Foltz 1958). The basic role of Se activity is its presence in catalytic sites of various selenoproteins. In eukaryotic cells, Se can be incorporated into 25 human and 24 rodent selenoproteins during translation, as selenocysteine (Sec), 21 st aminoacid, which is unique for essential trace elements incorporation (Hesketh 2008). Selenoproteins may perform various functions in humans, including antioxidant action (e.g., glutathione peroxidases), transport and storage of $\mathrm{Se}$ (selenoprotein $\mathrm{P}$ ), redox signaling (thioredoxin reductases), thyroid hormone metabolism (iodothyronine deiodinases), protein folding (e.g., selenoprotein $15 \mathrm{kDa}$ ), and others (Table 1).

The role of Se and selenoproteins in human health and diseases has been intensively studied with special attention on the determination of relevant biomarkers of Se status. The measured Se status depends mainly on dietary intakes. In the diet, Se is largely found in animal foods, and to a lesser extent also in plants, which indicates large individual differences in Se intake, associated with dietary menu composition, but also with the origin of food, which can be grown (plants) or bred (animals) on soils with different $\mathrm{Se}$ content (Gromadzinska et al. 2008). In humans, dietary Se 
Table 1 Human selenoproteins

\begin{tabular}{|c|c|c|c|}
\hline Function & Abbreviations & Selenoprotein & Cellular; tissue localization \\
\hline \multirow[t]{8}{*}{$\begin{array}{l}\text { Antioxidant } \\
\text { enzymes }\end{array}$} & $\mathrm{GPx} 1^{\mathrm{a}}$ & $\begin{array}{l}\text { Cytosolic glutathione peroxidase } \\
\text { (GPx) }\end{array}$ & Cytosol, mitochondria; widely expressed \\
\hline & $\mathrm{GPx} 2^{\mathrm{a}}$ & Gastrointestinal GPx & Cytosol, ER; gastrointestinal tissue, liver \\
\hline & GPx3 & Plasma GPx & $\begin{array}{l}\text { Secreted; plasma, extracellular fluid, liver, kidney, heart, lung, } \\
\text { thyroid, gastrointestinal tissue, breast }\end{array}$ \\
\hline & $\mathrm{GPx} 4^{\mathrm{a}}$ & Phospholipid hydroperoxide GPx & Cytosol, mitochondria, nucleus; widely expressed, testes \\
\hline & GPx6 & Olfactory GPx & Unknown; embryo and oilfactory epithelium \\
\hline & SelK & Selenoprotein K & $\mathrm{ER}$, membrane protein \\
\hline & SelR & $\begin{array}{l}\text { Selenoprotein R; methionine } \\
\text { sulfoxide reductase B1 }\end{array}$ & Cytosol, nucleus; widely expressed \\
\hline & SelW & Selenoprotein W & $\begin{array}{l}\text { Cytosol; widely expressed, brain, colon, heart, skeletal muscle, } \\
\text { prostate }\end{array}$ \\
\hline $\begin{array}{l}\text { Transport and } \\
\text { storage of Se }\end{array}$ & SepP1 ${ }^{\mathrm{a}}$ & Selenoprotein P & Secreted, cytosol; plasma, widely expressed, brain, liver, testes \\
\hline \multirow[t]{3}{*}{ Redox signaling } & $\operatorname{TrxR}^{\mathrm{a}}$ & Thioredoxin reductase, type I & Cytosol, nucleus; widely distributed \\
\hline & TrxR2 & Thioredoxin reductase, type II & Mitochondria; widely distributed \\
\hline & TrxR3 & Thioredoxin reductase, type III & Cytosol, ER, nucleus; testis-specific \\
\hline \multirow{3}{*}{$\begin{array}{l}\text { Thyroid hormone } \\
\text { metabolism }\end{array}$} & DIO1 & Iodothyronine deiodinase, type I & Membrane protein; kidney, liver, thyroid, brown adipose tissue \\
\hline & $\mathrm{DIO} 2$ & Iodothyronine deiodinase, type II & $\begin{array}{l}\text { ER, membrane protein; thyroid, central nervous system, brown } \\
\text { adipose tissue, skeletal muscle }\end{array}$ \\
\hline & DIO3 & Iodothyronine deiodinase, type III & Membrane protein; placenta, central nervous system, fetus \\
\hline \multirow[t]{4}{*}{ Protein folding } & Sep $15^{\mathrm{a}}$ & Selenoprotein $15 \mathrm{kDa}$ & ER lumen \\
\hline & SelN & Selenoprotein $\mathrm{N}$ & ER membrane; widely expressed \\
\hline & SelM & Selenoprotein M & ER lumen \\
\hline & $\mathrm{SelS}^{\mathrm{a}, \mathrm{b}}$ & Selenoprotein $S$ & ER, membrane protein; widely expressed \\
\hline Sec synthesis & SPS2 & Selenophosphate synthetase & Cytosol \\
\hline \multirow[t]{5}{*}{ Unknown } & SelH & Selenoprotein H & Nucleus; widely expressed \\
\hline & SelI & Selenoprotein I & Transmembrane \\
\hline & $\mathrm{SelO}$ & Selenoprotein $\mathrm{O}$ & Unknown \\
\hline & SelT & Selenoprotein $\mathrm{T}$ & ER membrane \\
\hline & SelV & Selenoprotein V & Testes \\
\hline
\end{tabular}

Adapted from Papp et al. (2007), Shchedrina et al. (2010), Fairweather-Tait et al. (2010)

${ }^{a}$ Genetic polymorphism associated with cancer

b Inflammatory disease

intakes also vary geographically from low to high Se areas. Well documented Se deficiency health effects-Keshan and Kashin-Beck (KBD) diseases, associated with muscle disorders, were found in a broad zone running from northeast to southwest China, from the border of Heilongjiang to the Yunnan province, where concentration of $\mathrm{Se}$ in the soil was very low (Oldfield 1999). In European countries, dietary Se intake is lower than that observed in USA, mainly due to low Se soils. Recommended dietary intake (RDI) value of Se for adults in USA and Europe is $55 \mu \mathrm{g} /$ day. Tolerable upper intake level determined by the US Food and Nutrition Board (NAS 2000) is $400 \mu \mathrm{g} / \mathrm{day}$, while that determined by the Scientific Committee on Food in Europe is $300 \mu \mathrm{g} /$ day (EFSA 2008). Average intake of Se by European population ranges from 27 to $70 \mu \mathrm{g}$ per day (EFSA 2008), which is insufficient to meet the daily requirement. Clinical signs of marginal Se deficiency in Europe have not been observed or documented yet. However, it should be noted that several groups of healthy individuals may be specially prone to Se deficiency, which includes breast-fed neonates, pregnant women, and elderly people (Bellinger et al. 2009).

The relationship between Se level and health effects is represented by a $U$-shaped curve that suggests health pathologies associated with Se deficiency as well as its excess (Ip 1998; Jablonska et al. 2008). The altered Se status resulting from insufficient Se intake is very often associated with different diseases, including immune diseases, cardiological diseases, and cancer. On the other hand, recent studies have indicated that long-term high 
dietary Se supply seems to be related to the risk of type 2 diabetes, amyotrophic lateral sclerosis, and some types of cancer (Bellinger et al. 2009; Nogueira and Rocha 2011).

Although Se levels in blood and blood compartments are easily accessible markers of human Se nutritional status, $\mathrm{Se}$ level itself does not reflect its functional significance. Plasma or serum Se, very often used in various Se investigations, reflects rather short-term Se status, while platelet, leukocyte, and erythrocyte Se reflects its longer-term status. Two best known selenoprotein biomarkers that have been widely used in discriminating of Se status are as follows: plasma selenoprotein P (SePP1) level and plasma glutathione peroxidase (GPX3) activity. In healthy humans, plasma $\mathrm{Se}$ is incorporated as $\mathrm{Sec}$ in two selenoproteins: SePP1 (40-70\%), GPx3 (20-40\%), while 6-10\% of Se is bound to albumin in the form of selenomethionine, through the replacement of methionine. Free Se accounts for less than $1 \%$ of total plasma Se (Vincent and Forceville 2008). These biomarkers generally reflect the major sources of human body Se, because GPx3 and SePP1 are the unique secreted selenoproteins. GPx3 is mainly synthesized in kidney, where it is produced by the cells of proximal tubular ephitelium and by parietal cells of Bowman's capsule and then it is released into the plasma (Gromadzinska et al. 2008; Reeves and Hoffmann 2009). Mammalian SePP1 that contains multiple Sec residues (10 Sec residues in humans and rodents) is synthesized in liver and then secreted into the blood and transported to other tissues. Therefore, SePP1 serves as Se transporter and Se body distribution controller. Recently, specific apolipoprotein E receptor-2 (ApoER2) for SePP1 uptake in brain and testis and ApoER2 homolog-megalin-for SePP1 uptake in kidney proximal tubule epithelial cells were found, suggesting receptor-mediated uptake of Se in these organs (Burk and Hill 2009).

There is a growing interest in the use of transcripts level as a molecular biomarker with special regard to whole blood. Since early 1990s, research on this new molecular biomarker of Se status has been extended to Se studies in rodents, showing prioritization of preservation and degradation of specific selenoprotein mRNA under Se deficiency and under conditions of adequate and enhanced supply (Bermano et al. 1995). The observed hierarchy of the expression of selenoprotein mRNA in response to dietary Se supply has indicated the order of selenoproteins, some of them dramatically affected under Se deficiency or its excess, and others only marginally. Selenoprotein transcripts analysis in the blood gives an opportunity to obtain genomic fingerprints, in response to $\mathrm{Se}$ status, but also reflect an impact of genetic polymorphism of selenoproteins. In a few recent human studies, selenoprotein gene expression level in circulating human blood leukocytes was used as a longer term Se status indicator. Some other studies have been focused on the role of Se incorporation during selenoprotein synthesis under different Se dietary status in humans (Pagmantidis et al. 2008; Ravn-Haren et al. 2008a, b; Reszka et al. 2009; Sunde et al. 2008).

Levels of Se for optimization of SePP1 concentration and GPx3 activity in plasma have been determined in people living in Se-adequate areas. However, these biomarkers may be unsuitable under conditions of high Se status, because of plateau levels obtained at such concentrations. On the other hand, Se levels in blood of individuals living in low Se areas with low Se intake may be insufficient to ensure maximal activity and/or concentration of selenoproteins GPx3 and SePP1 (Thomson 2004). Therefore, determination of Se intake and Se status in humans with variable Se supply seems to be important in assessing the most sensitive Se status biomarkers. In this review, we present recent findings regarding molecular Se biomarkers, based on rodent and human studies.

\section{Traditional biomarkers of Se status}

Se status measured by serum/plasma Se, plasma SePP1 concentration and plasma GPx3 activity may differently respond to Se supplementation, which can give information about low, adequate, and high Se dietary intake. Since several years, it has been generally accepted that selenoprotein level and/or activity may be more useful in determining Se status than Se itself. Two human selenoproteins, GPx3, and SePP1 are believed to be good nutritional Se biomarkers in humans. Studies on Se-deficient populations showed that full expression of SePP1 required larger intake of Se than did GPx3 activity (Xia et al. 2005, 2010). Therefore, setting RDI values for Se intake was based on their assessment of the amount of Se required to achieve optimal activity or two-thirds optimal activity of GPx3 in plasma to meet the requirements of people living in low, adequate, and high Se areas (Rayman 2004).

Different chemical forms of dietary Se from animal foods and plants, such as selenite, selenocysteine, and selenomethionine, are involved in metabolic pathways to form selenide. Selenide is then transformed into Sec for selenoprotein biosynthesis or may be methylated to the main metabolite - selenosugar and then excreted with urine (Rayman 2004). Selenomethionine is a major form of organic selenium in plant foods, similarly like seleniumenriched yeast in Se supplements. Because of different metabolism of Se compounds in organism, absorption of Se from different organic and inorganic Se food sources, and Se supplements, their incorporation in selenoprotein and urinary Se excretion may vary in humans. Studies on North Americans (Burk et al. 2006) and Se-deficient Chinese subjects (Xia et al. 2005) clearly indicate that Se in the 
form of selenomethionine is more easily absorbed than selenite. Absorption of Se from yeast was greater than this inorganic form but less than selenomethionine. Interestingly, plasma Se seemed to reflect selenomethionine content of yeast but not the other yeast Se forms, indicating its effective bioavailability (Burk et al. 2006).

In several populations in suboptimal Se areas in Europe, China, New Zealand, concentration of SePP1 could not reach the plateau because of low Se daily intake, suggesting that Se nutritional requirements had not been achieved (Xia et al. 2005). In Se-deficient Chinese subjects, different plateaus reached by plasma GPx3 activity and plasma SePP1 level indicates that the latter is a better indicator of Se status in humans, because a larger intake of Se has been required for optimized SePP1 concentration than did GPx3 activity (Xia et al. 2010). In addition to GPx3 activity, plasma SePP1 level may be a suitable Se status and Se intake biomarker in individuals from low Se populations with additional supply of Se. In populations living in high Se areas, e.g., Australia (Queensland), USA, and Canada (central states and provinces) (Oldfield 1999), where GPx3 activity and SePP1 level in plasma can be optimized by dietary Se supply, plasma Se seems to reflect Se status and Se intake (Burk et al. 2006).

\section{Molecular biomarkers of Se status}

The unique conserved stem-loop Sec insertion sequence (SECIS) in the $3^{\prime}$-untranslated region of mammalian selenoprotein mRNA is essential for the recognition of UGA as a codon for Sec. Sec may be synthesised from different Se dietary sources: selenomethionine, selenocysteine or selenite, Se compounds which are further metabolized to selenide and then in the presence of selenophosphate synthetase 2 to selenophosphate This process requires specific Sec tRNA[Ser]Sec, several translation factors like $\mathrm{SBP}$, EFSeC, and others that serve to distinguish between UGA codons designated for Sec from those terminating translation. Sec synthesis occurs directly on its Sec tRNA[Ser]Sec, initially carrying a serine residue, which serves as an acceptor for selenophosphate. Maturation of Sec tRNA[Ser]Sec requires methylation, and two isoforms of methylated and unmethylated Sec tRNA[Ser]Sec are observed. Se supplementation is known to modulate the relative ratio between these two isoforms and promote the methylation of Sec tRNA[Ser]Sec. (Hatfield and Gladyshev 2002; Hesketh and Villette 2002; Jameson and Diamond 2004; Schomburg and Schweizer 2009; SmallHoward and Berry 2005).

It has been observed that the alterations in selenoprotein activity and concentration during Se depletion and repletion are accompanied by changes in the mRNA level.
Under severe Se deficiency, this microelement is accumulated mainly in the brain and endocrine tissues, where elevated expression and activity of phospholipid glutathione peroxidase $(\mathrm{GPx} 4)$ and thyroxine deiodinase (DIO) were observed, indicating the biological importance of these selenoproteins (Schomburg and Schweizer 2009). According to rodent studies, the main selenoproteins that are resistant to dietary Se changes are GPx4 and DIO; thioredoxine reductases (TrxR) and SePP1 are moderately sensitive, while GPx1, SelW, SelH are very sensitive to low Se supply (Reeves and Hoffmann 2009; Sunde 2010). Several in vitro and in vivo studies have shown that, under Se deficiency, the degradation of selenoprotein mRNA occurs through NMD (nonsense codon-mediated mRNA decay) (Moriarty et al. 1998; Weiss and Sunde 1998). Due to specific nucleotide sequences and preferential binding of SBP2 translation factor, 14 of 25 human selenoprotein mRNAs may be sensitive to NMD-based degradation under low Se supply (Squires et al. 2007). However, a study on rodents, suggesting regulation of selenoprotein expression, irrespective of this hypothetical preference, indicates that also other mechanisms concerning differences in the expression efficiency of some selenoproteins under Se deficiency must exist (Sunde et al. 2009). In addition, methylated Sec tRNA[Ser]Sec supports the biosynthesis of selenoproteins GPx1, GPx3, SelR, SelT, sensitive to low Se supply, while other selenoproteins, like TrxR1 and GPx4, moderately sensitive and resistant to low Se supply, require unmethylated isoform (Schomburg and Schweizer 2009).

\section{Selenoprotein transcripts in rodents}

An attempt to answer the question whether selenoprotein gene expression may be used as a biomarker of Se status was presented for the first time by Roger A. Sunde's team. Seppl mRNA shows the highest expression in rat testes and liver, while Gpx3 mRNA expression is highest in rat kidney (Evenson et al. 2004), which is in agreement with observed selenoprotein activity and protein expression in these organs. Therefore, these molecular biomarkers may be also useful for the determination of Se status as well as for establishing physiological requirements of $\mathrm{Se}$ for adequate selenoprotein gene expression. These authors found also that, in rodents, Gpxl, Selh, and Selw mRNA levels were highly regulated by Se status (Sunde 2010). Analysis of hierarchical regulation of different selenoprotein mRNAs by Se status indicates that Gpxl transcripts level is the best and adequate molecular biomarker in rats, because dietary Se deficiency similarly decreased Gpxl mRNA in blood and liver. By way of comparison, decrease in $\mathrm{Se}$ supply in diet did not affect Gpx4 mRNA in liver and blood. Gpxl mRNA expression in rat liver was the highest, 
while in blood, expression of this selenoprotein was at 4th place in tissue rank, comparable with the expression in heart and kidney. Interestingly, other investigated selenoprotein transcripts also presented distinct expression pattern across the tissues and it was found that Gpxl, Gpx3, Gpx4, Sepp1, Trxrl expression in blood were comparable with those observed in the major organs (Evenson et al. 2004).

Regulation of selenoprotein mRNA under Se dietary deficiency presents diversity, from dramatic decrease in Gpxl mRNA to lack of changes for Gpx4 mRNA. Low selenoprotein mRNA observed in rodents reflect depletion of Se supply in the diet, and adequate Se supply regulates expression level very efficiently. It has been found that at least half of the dietary Se necessary to provide plateau for enzymatic activity or protein expression is adequate to provide plateau for mRNA expression in liver, muscle, and kidney of rats (Barnes et al. 2009). With increasing Se level, selenoprotein mRNA level is expressed as hyperbolic curve presenting plateau breakpoints at low Se supply for majority of selenoproteins. Minimal Se requirement for growing rats was $0.1 \mu \mathrm{g} \mathrm{Se} / \mathrm{g}$ diet based on liver Se, liver, and RBC GPx1 activity. Slightly lower dietary Se requirements based on plasma GPx3 activity, liver TrxR activity, and liver and kidney GPx3 activity were observed. Based on dose-response curves for selenoprotein mRNA indifferent tissues, the minimum dietary requirements were lower than for physiological Se biomarkers, ranging between 0.04 and $0.06 \mu \mathrm{g} \mathrm{Se} / \mathrm{g}$ diet in liver and kidney and was between 0.03 and $0.05 \mu \mathrm{g} \mathrm{Se} / \mathrm{g}$ diet in muscles. Besides, it has been assumed that it is not feasible to use selenoprotein mRNA in rat tissues as a biomarker for super-nutritional Se level (up to $0.8 \mu \mathrm{g} \mathrm{Se} / \mathrm{g}$ diet). These experiments evidently suggest that marginal level of Se in diet is able to increase selenoprotein mRNA to adequate level in rats and mice, what may suggest common mechanism in regulation of selenoprotein mRNA expression by dietary Se supply (Barnes et al. 2009). Recently, Sunde (2010) proposed the panel of molecular biomarkers, which could be useful for the assessment of selenium status in rats and might be effective as the traditional biomarker panel in rat tissues. Gpx1, Gpx3, Selt, and Selw mRNA panel of transcripts level was significantly correlated with liver Se concentration, Gpxl and Selk mRNA were associated with liver Gpxl activity, while Sepw and Selk mRNA reflected kidney selenium status, and Gpxl, Sepw, Txnrdl transcripts level correlated with Gpxl kidney activity.

Interestingly, recent studies suggest that regulation of selenoprotein mRNA by Se dietary status is not a general phenomenon in rodents. It was found that in mice, majority of analyzed selenoprotein transcripts in liver and kidney were not significantly regulated by Se deficiency (Sunde et al. 2009). It has been found that minimum $\mathrm{Se}$ requirement of the turkey is higher than for rodents (Sunde and Hadley 2010). Dietary requirement was decreased at least $50 \%$ in old rats as compared to requirements of young animals (Sunde and Thompson 2009). Expression of the majority of selenoprotein mRNA in testis, except for Gpxl, Sepp1, Sepw, and also Apoer2, was not regulated by dietary Se status (Schriever et al. 2009). The mRNA abundances of the 12 selenoprotein genes in thyroid and pituitary of young pigs were resistant to increasing Se dietary supply in diet and also Se deficiency, but not in liver, where NMD under Se deficiency was observed for Gpxl, Sepw, Sepn, Txrd1. The testicular mRNA of Txnrdl and Sep15 were decreased by increasing dietary Se supply, indicating that high Se status may be associated with a decrease in selenoprotein mRNA transcripts level (Zhou et al. 2009).

Differences in dietary minimal Se supply required for maximal mRNA expression and/or selenoenzyme activity suggest differential regulation depending on the type of selenoprotein as well as tissue. Therefore, findings regarding ranking of different selenoproteins synthesis within tissue and also different distribution of selenoproteins in various tissues should be considered in establishing a universal Se status biomarker (Sunde 2010).

\section{Selenoprotein transcripts in humans}

In 2003, the panel of experts in UK Food Standards Agency has issued specific research recommendations after evaluation of current knowledge regarding the assessment of Se status, including further development of functional biomarkers (Elsom et al. 2006). Recently, a special emphasis is laid on the application of population of circulating white blood cells (WBC) transcriptome patterns in nutritional studies. Transcriptomics studies suggest that genes with various functional annotations can be significantly expressed in WBC (Visvikis-Siest et al. 2007). Gene expression patterns may be also useful to define biological processes associated with human health and disease. However, only a few studies assessing selenoprotein gene expression in various populations of circulating WBC in humans have been conducted so far (Table 2).

Human studies have not confirmed the hypothesis that selenoprotein transcript level in circulating leukocytes and in whole blood may be the reliable biomarker of Se status in population with adequate (Sunde et al. 2008) and suboptimal (Reszka et al. 2009) baseline Se level in plasma (Fig. 1). After short-term $100 \mu \mathrm{g}$ sodium selenite supplementation, healthy subjects from the SELGEN population were chosen at random for microarray analysis of RNA isolated from lymphocytes. The greatest changes after Se supplementation were observed for genes that encode proteins functioning in protein biosynthesis. Up-regulation 
Table 2 Human studies on selenoprotein molecular biomarkers

\begin{tabular}{|c|c|c|c|}
\hline Study & $\begin{array}{l}\text { Baseline daily } \\
\text { Se intake }(\mu \mathrm{g}) \\
\text { Mean } \pm \mathrm{SD}\end{array}$ & $\begin{array}{l}\text { Baseline plasma } \\
\text { Se level }(\mathrm{ng} / \mathrm{ml}) \\
\text { Mean } \pm \mathrm{SD}\end{array}$ & $\begin{array}{l}\text { Selenoprotein gene expression } \\
\text { in WBC }\end{array}$ \\
\hline $\begin{array}{l}\text { UK, SELGEN study, } n=39 \text {, both } \\
\text { sexes, } 6 \text { week. Se intervention } \\
\text { (Pagmantidis et al. 2008) }\end{array}$ & Not presented & $93.9 \pm 1.7$ & $\begin{array}{l}\text { Lymphocyte } \\
\text { Up-regulation: } \\
\text { SPS1-1.15 (1.06-1.23) } \\
\text { SelK-1.11 (1.04-1.19) } \\
\text { Sep15-1.11 (1.02-1.20) }\end{array}$ \\
\hline $\begin{array}{l}\text { Denmark, } n=20,18-40 \text { years. } \\
\text { old men, } 1 \text { week Se intervention } \\
\text { (Ravn-Haren et al. 2008a) }\end{array}$ & $49.8 \pm 13.6$ & $113.2 \pm 12.2$ & $\begin{array}{l}\text { Leukocyte } \\
\text { No effect: } G P x 1, \operatorname{Tr} x R 1 \text { SePPl }\end{array}$ \\
\hline $\begin{array}{l}\text { Denmark, PRECISE pilot study, } \\
n=105 \text {, both sexes, up to } \\
5 \text { years. Se intervention } \\
\text { (Ravn-Haren et al. } 2008 \text { b) }\end{array}$ & $49.8 \pm 13.6$ & $93 \pm 11.2$ & $\begin{array}{l}\text { Leukocyte } \\
\text { No effect: } G P x l\end{array}$ \\
\hline $\begin{array}{l}\text { UK, } n=39 \text {, both sexes, } 28 \text { week. } \\
\text { Se intervention (Sunde et al. 2008) }\end{array}$ & $48 \pm 14$ & $89.2 \pm 12.5$ & $\begin{array}{l}\text { Whole blood } \\
\text { No effect and no association between } \\
\text { Se status and GPxl, GPx3, } \\
\text { GPx4, SePP1, SepW, SepH }\end{array}$ \\
\hline $\begin{array}{l}\text { Poland, } n=47 \text { healthy men } \\
\text { (Reszka et al. 2009) }\end{array}$ & $24.2 \pm 17.4$ (unpublished data) & $54.3 \pm 14.6$ & $\begin{array}{l}\text { Leukocyte } \\
\text { No association between Se } \\
\text { status and GPx1, GPx3, } \\
\text { SePP1, Sep } 15\end{array}$ \\
\hline
\end{tabular}

of selenoprotein $\mathrm{K}(\mathrm{SelK})$ and selenoprotein $15 \mathrm{kDa}$ (Sep15) after Se supplementation was observed, indicating that only a small number of selenoprotein encoding genes was altered by different Se dietary supply (Pagmantidis et al. 2008). Results obtained by Ravn-Haren et al. (2008a, b) also indicated lack of Se impact on selenoprotein mRNA expression in WBC after short- and long-term Se supplementation. After 1 week of supplementation with nonorganic Se or organic form among healthy young Danish men, there were no differences in mRNA expression of GPxl, TrRl, and SePl in leukocytes (Ravn-Haren et al. 2008a). Similarly, after 5 years of Se-enriched yeast supplementation of the PRECISE Danish subjects, there were no differences in the expression of GPxl in circulating WBC (Ravn-Haren et al. 2008b). Moreover, selenoprotein expression level in whole blood was not significantly associated with Se status measured in the blood and $\mathrm{Se}$ supply measured according to dietary questionnaire in UK population (Sunde et al. 2008). The baseline Se level in UK and Danish populations was relatively high as compared to other European populations and it approximately averaged $100 \mathrm{ng} / \mathrm{ml}$ in plasma (Table 2). Lack of changes in selenoprotein mRNA levels after Se supplementation indicates that protein synthesis may already be saturated in leukocytes at such sufficient Se concentration. In the small group of 47 healthy Polish individuals, plasma Se levels were below the level required to optimize plasma GPx3 activity $(54.3 \pm 14.6 \mathrm{ng} / \mathrm{ml})$. However, no relationship between serum Se and GPxl, GPx3, SePP1, and Sep15 mRNA transcripts level, as well as GPx1 and GPx3 activities and selenoprotein mRNA expression was found (Reszka et al. 2009). Daily Se intake in studied European groups was lower than RDI (55 $\mu \mathrm{g} /$ day) for adults in Europe and US. The observed hyperbolic curve describing the relationship between selenoprotein mRNA expression and dietary supply in rodents suggests that suboptimal Se status in humans may be sufficient for selenome transcription machinery. Interestingly, GPx1, GPx3, SePP1, and Sep15 selenoprotein mRNA expression in circulating blood leukocytes was significantly positively correlated, indicating similar regulation of expression in circulating blood leukocytes. Although significant correlations were found between several selenoprotein mRNAs in the circulating blood leukocytes of healthy individuals, no correlation was found in the blood of bladder cancer patients, which may suggest an alteration of selenoprotein synthesis during carcinogenesis (Reszka et al. 2009).

\section{Modifiers of selenium status}

Functional significance of blood Se status is mainly related to selenoprotein activity in specific tissues. Therefore, both traditional and molecular biomarkers of Se status measured in different human tissues, except for controlled Se intake, may depend on additional major modifiers, like health 
Fig. 1 Correlation between plasma Se and GPx3 transcripts level in population with adequate-British (whole blood) (Sunde et al. 2008) (a) and suboptimal-Polish (leukocyte) (Reszka et al. 2009) (c) Se level and correlation between plasma Se level and SePP1 transcripts level in population with adequateBritish (whole blood) (b) and suboptimal-Polish (leukocyte) (d) Se level $\mathbf{a}$ and $\mathbf{b}$ reproduced with permission from Sunde et al. (2008)
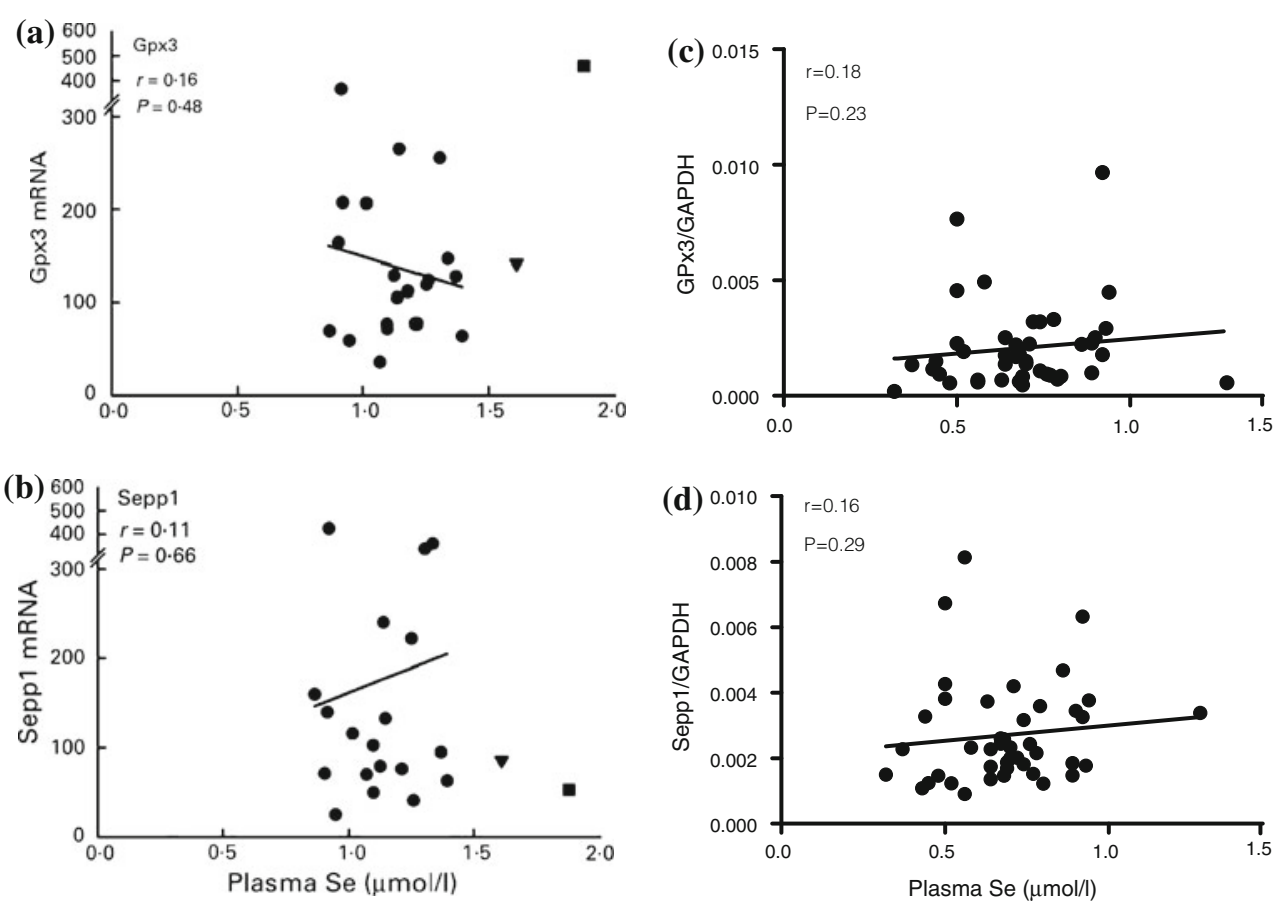

status (e.g., endocrine and immunological status), interindividual variations (age, sex, genetic polymorphism), environmental exposure, diet, medication, etc. (Fig. 2). Therefore, establishing the Se status and intake which would be optimal for human health seems to be very difficult, especially in many populations experiencing suboptimal Se supply, including Europe. Epidemiological and animal studies clearly indicate that biological effects of $\mathrm{Se}$ are sex-specific, which may be associated with endocrine regulation and also immunological status. It has been suggested that cancer risk in men is more profoundly influenced by Se status than in women (Waters et al. 2004). Selenoprotein gene expression displays sexual dimorphism in various organs of females and males, e.g., Se status was linked to male fertility due to GPx4 function during spermatogenesis (Schomburg and Schweizer 2009). Sex-specific selenoprotein expression pattern may vary or be sustained with age. In the SELGEN study, an effect of Se supplementation was associated with GPx4 genetic polymorphism in a sex-specific manner (Meplan et al. 2008). Plasma Se level is not likely to accurately reflect tissue Se status or selenoenzyme activity and level. There are several confounders potentially influencing Se level. Liver and kidney diseases can cause alterations of SePP1 (Li et al. 2007) and GPx3 levels (Zachara et al. 2006). Se metabolism may be altered in different health pathologies, e.g., in patients with inflammatory diseases. During critical illness, like sepsis, acute phase response, and other immunological disturbances, serum Se status may be lower and insufficient to support organ function. In systemic inflammatory response syndrome patients, serum Se concentration was significantly lower (Vincent and Forceville 2008). Tobacco smoking or occupational exposure can also increase dietary requirement of Se. It is generally agreed that smoking can decrease the activity of antioxidative selenoproteins, probably due to formation of complexes of Se with cadmium (Ellingsen et al. 2009).

In human population, there is a large individual variation in response to Se supplementation, appearing to be unrelated with the baseline Se status (Brown et al. 2000), which may clearly indicate occurrence of selenoprotein gene variants. Recent human studies indicate that selenium status, measured as body Se, as well as SePP1 plasma level and GPx activity, may be significantly influenced by genetic polymorphism of specific selenoproteins, including GPx1 (rs1050450) (Jablonska et al. 2009), SePP1 (rs7579, rs3877899) (Meplan et al. 2007, 2009), GPx4 (rs713041) (Meplan et al. 2008). The impact of SePPl variations in codon 234, associated with Ala to Thr change (rs3877899) and $G$ to $A$ transition within 3' untranslated region (UTR) of SePP1 mRNA (rs7579), resulted in the alteration of Se status before as well as after Se supplementation (Meplan et al. 2007). These polymorphisms also influence proportion of two SePP1 50 and $60 \mathrm{kDa}$ isoforms in plasma, which was proposed as the modulation factor of $\mathrm{Se}$ incorporation during selenoprotein synthesis (Meplan et al. 2009). Se availability modulated by SePPl variants, resulting in difference in isoform pattern was restricted just to males. Interestingly, a possible impact of gender was observed for functional significance of GPx4 genetic polymorphism. Single nucleotide polymorphism in $3^{\prime}$-UTR of GPx4 mRNA, associated with C to T change (rs713041) 
Fig. 2 Biomarkers of selenium status in humans. A scheme of traditional and molecular biomarkers measurements with the impact of potential modifiers (physiological, environmental, genetic). The optimal selenium biomarker should reflect all putative egzo- and endogenous factors which can modulate selenium bioavailability, metabolism and selenoprotein transcription, biosynthesis, transport, activity and function. A type of measurement used for the determination of selenium status should be also considered

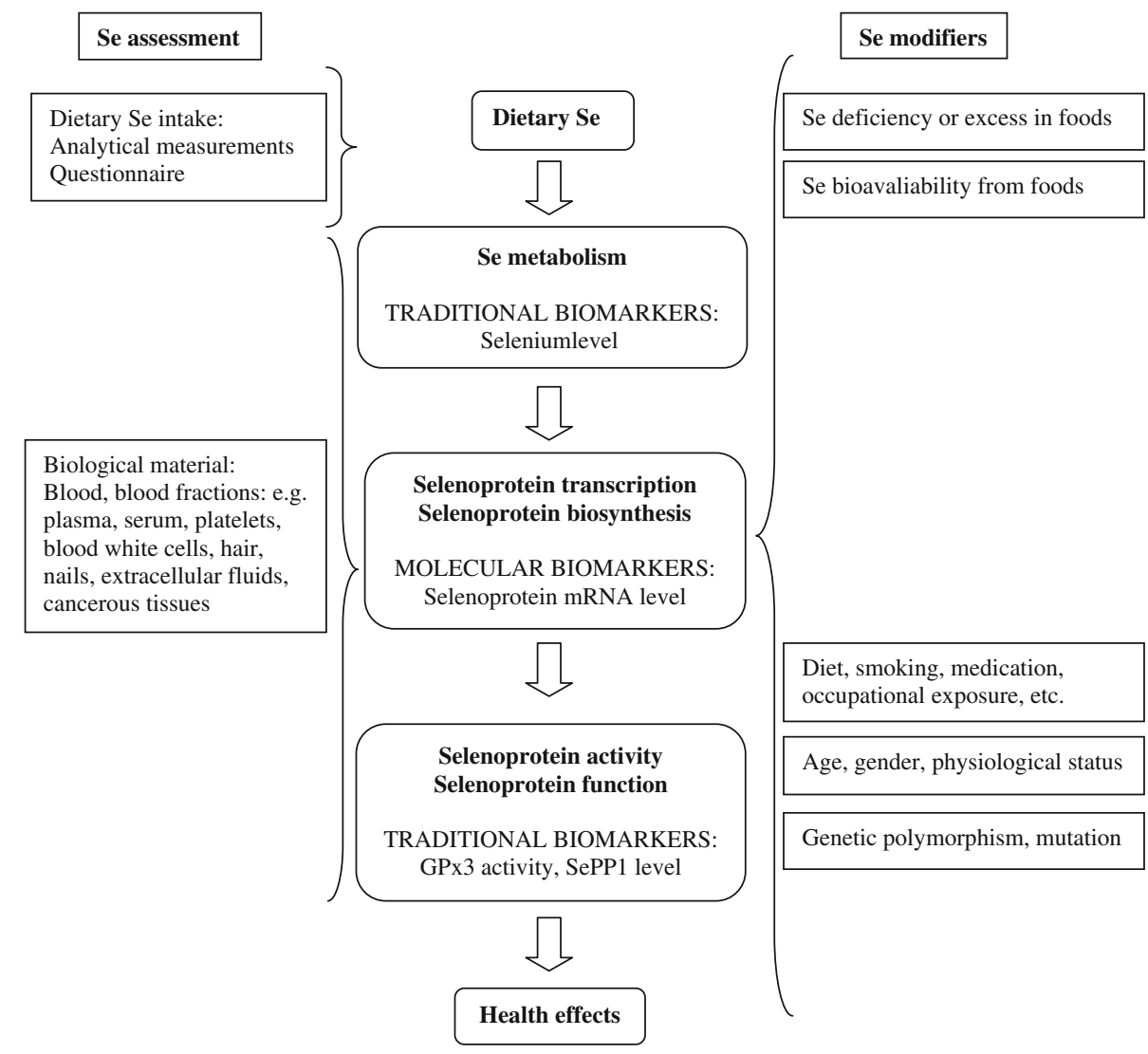

influenced the level of GPx4 in lymphocytes, and also other selenoproteins; however, this effect was more evident in females (Meplan et al. 2008). One may hypothesize that separate molecular mechanisms for GPX4 synthesis in testes and high dietary Se requirements in males override genetic polymorphism in GPx4 and SePP1. Recent findings suggest also that the association between GPx1 activity and Se concentration, analyzed separately for each GPxl Pro198Leu (rs1050450) genotype group, was the highest for Pro/Pro and the lowest for Leu/Leu genotype, suggesting different response of GPx1 activity to Se. This also points to the importance of the genetic background in the assessment of the Se status with the use of selenoprotein biomarkers such as GPx1 activity (Jablonska et al. 2009).

Adaptation of humans to suboptimal dietary Se supply with low Se level was observed by Finley et al. (1999) in healthy study participants living in New Zealand, where people consume less Se than suggested by the RDI of $55 \mu \mathrm{g} /$ day. Se level in blood of individuals living in low Se areas and with low Se intake may be insufficient for maximal activity of GPx3 and level of SePP1 in plasma. However, supplementation had no effect on Se status in platelets and erythrocytes, which can be regarded as indicative of long-term $\mathrm{Se}$ intake. Interestingly, high retention of stable ${ }^{74} \mathrm{Se}$ isotope in placebo individuals was observed as compared to individuals supplemented with
$30 \mu \mathrm{g}$ Se daily for 5 months, suggesting maintenance of critical Se pool in the human body and adaptation to low Se cal requirements of $\mathrm{Se}$ are lower than recommended in humans, but enabling selenoprotein synthesis. Besides, in patients with diseases like KBD and cancer, where low Se level in blood is observed, down-regulation of specific selenoprotein gene expression was found to occur in circulating WBC. Significant down-regulation of SePPl mRNA level was observed in Han Chinese with KBD (Sun et al. 2010). In Caucasian bladder cancer male patients, SePP1, GPx1, GPx3, Sep15 mRNA levels were lower than in the control group (Reszka et al. 2009). Preferential incorporation of Sec into selenoprotein mRNA in circulating blood leukocytes under relatively low Se supply merits investigations intended to identify potential sensitive and resistant selenoproteins under Se deficiency in humans.

\section{Concluding remarks}

While the levels of Se required for optimization of SePP1 and GPX3 activities in plasma are well known, estimating Se level that is required for maximal selenoprotein gene expression in humans requires further research. According status by adjusting of its secretion. Therefore, physiologi- 
to rodents studies and studies among humans, which have been discussed in this review, it appears that suboptimal Se intake may be sufficient to achieve selenoprotein mRNA expression and molecular requirements of Se are lower than the established recommended dietary intake in humans-55 $\mu \mathrm{g} /$ day (EFSA 2008; NAS 2000). It should be noted that the expression of individual selenoprotein mRNA may be not always linked with protein expression. Therefore, since we have not understood the transcriptional and translational link of selenoprotein under different $\mathrm{Se}$ supply in different human tissues, biological functionality of selenoprotein could be recognized only at protein level.

Preferential incorporation of Se into selenoproteins in rodents was observed even at suboptimal dietary Se level. In rats, minimum Se requirements for achieving a plateau of selenoprotein mRNA expression have been low, reached plateau at half of the dietary Se concentration required for maximal activity of GPx1, GPx3, and maximal level of SePP1 in different tissues (Barnes et al. 2009). Therefore, in rodents, selenoprotein mRNA expression does not seem to be a good indicator for Se status. Hypothetically, in almost all populations, even in those with low and moderate Se intake and Se level in blood compartments, selenoprotein encoding gene expression might reach the plateau levels and serve as a sensitive functional biomarker of Se status. However, it should be noted that regulation of selenoprotein gene expression, metabolic pathways, and responses to Se interventions in animals may differ from those in humans. None of the human studies conducted so far has indicated that selenoprotein mRNA in the whole blood and blood cells may be a good indicator of Se status in humans (Pagmantidis et al. 2008; Ravn-Haren et al. 2008a, b; Reszka et al. 2009; Sunde et al. 2008). Low Se status may be adequate for proper regulation of selenoprotein transcription, but not for proper physiological activity of selenoproteins. Preferential incorporation of Se into selenoprotein and its optimal saturation at low dietary Se level does not provide adequate activities and selenoprotein expression in different tissues. Therefore, it may be suggested that functional rather than molecular biomarkers of Se are optimal indicators of its supply. It seems reasonable to conclude that SePP1 concentration in plasma may clearly indicate sub-optimal to optimal Se supply, because it can reflects functional significance of Se activity in organism. In over-optimal Se supply, where SePP1 level is optimized, plasma $\mathrm{Se}$ seems to reflect Se intake and achieved Se status.

All possible modifiers of Se status determined by means of biochemical and molecular techniques, including smoking, occupational exposure, diet, health parameters, sex, age, endocrine and immunological status, should be included, as these factors can potentially modulate selenoprotein gene expression. Impact of polymorphism of selenoproteins should be also included in complete analysis of Se requirements of individuals with different genotypes and haplotypes. Therefore, comprehensive intervention studies of circulating leukocyte selenoprotein transcript levels in a population with suboptimal and with adequate compared to over-optimal selenium status should be conducted.

Acknowledgments This work was financially supported by the Ministry of Science and Higher Education (grant 1978/B/P01/2009/37 and 1666/B/P01/2011/40) and NIOM internal grants IMP 1.3/2007, IMP $1.8 / 2009$. Part of this work was presented at 9th International Symposium on Selenium in Biology and Medicine. Selenium 2010, May 31-June 4 Kyoto, Japan.

Open Access This article is distributed under the terms of the Creative Commons Attribution Noncommercial License which permits any noncommercial use, distribution, and reproduction in any medium, provided the original author(s) and source are credited.

\section{References}

Barnes KM, Evenson JK, Raines AM et al (2009) Transcript analysis of the selenoproteome indicates that dietary selenium requirements of rats based on selenium-regulated selenoprotein mRNA levels are uniformly less than those based on glutathione peroxidase activity. J Nutr 139:199-206

Bellinger FP, Raman AV, Reeves MA et al (2009) Regulation and function of selenoproteins in human disease. Biochem J 422:11-22

Bermano G, Nicol F, Dyer JA et al (1995) Tissue-specific regulation of selenoenzyme gene expression during selenium deficiency in rats. Biochem J 311:425-430

Brown KM, Pickard K, Nicol F et al (2000) Effects of organic and inorganic selenium supplementation on selenoenzyme activity in blood lymphocytes, granulocytes, platelets and erythrocytes. Clin Sci 98:593-599

Burk RF, Hill KE (2009) Selenoprotein P-expression, functions, and roles in mammals. Biochim Biophys Acta 1790:1441-1447

Burk RF, Norsworthy BK, Hill KE et al (2006) Effects of chemical form of selenium on plasma biomarkers in a high-dose human supplementation trial. Cancer Epidemiol Biomarkers Prev 15:804-810

EFSA (2008) Scientific opinion of the panel on food additives, flavourings, processing aids and materials in contact with food (AFC) on a request from the commission on selenium-enriched yeast as a source for selenium. EFSA J 766:1-43

Ellingsen DG, Thomassen Y, Rustad P et al (2009) The time-trend and the relation between smoking and circulating selenium concentrations in Norway. J Trace Elem Med Biol 23:107-115

Elsom R, Sanderson P, Hesketh JE et al (2006) Functional markers of selenium status: UK Food Standards Agency workshop report. Br J Nutr 96:980-984

Evenson JK, Wheeler AD, Blake SM et al (2004) Selenoprotein mRNA is expressed in blood at levels comparable to major tissues in rats. J Nutr 134:2640-2645

Fairweather-Tait SJ, Collings R, Hurst R (2010) Selenium bioavailability: current knowledge and future research requirements. Am J Clin Nutr 91:1484S-1491S

Finley JW, Duffield A, Ha P et al (1999) Selenium supplementation affects the retention of stable isotopes of selenium in human subjects consuming diets low in selenium. Br J Nutr 82:357-360

Gromadzinska J, Reszka E, Bruzelius K et al (2008) Selenium and cancer: biomarkers of selenium status and molecular action of selenium supplements. Eur J Nutr 47(Suppl 2):S29-S50 
Hatfield DL, Gladyshev VN (2002) How selenium has altered our understanding of the genetic code. Mol Cell Biol 22:3565-3576

Hesketh J (2008) Nutrigenomics and selenium: gene expression patterns, physiological targets, and genetics. Annu Rev Nutr 28:157-177

Hesketh JE, Villette S (2002) Intracellular trafficking of micronutrients: from gene regulation to nutrient requirements. Proc Nutr Soc 61:405-414

Ip C (1998) Lessons from basic research in selenium and cancer prevention. J Nutr 128:1845-1854

Jablonska E, Gromadzinska J, Sobala W et al (2008) Lung cancer risk associated with selenium status is modified in smoking individuals by Sep 15 polymorphism. Eur J Nutr 47:47-54

Jablonska E, Gromadzinska J, Reszka E et al (2009) Association between GPx1 Pro198Leu polymorphism, GPx1 activity and plasma selenium concentration in humans. Eur J Nutr 48:383386

Jameson RR, Diamond AM (2004) A regulatory role for Sec tRNA[Ser]Sec in selenoprotein synthesis. RNA 10:1142-1152

Li CL, Nan KJ, Tian T et al (2007) Selenoprotein P mRNA expression in human hepatic tissues. World $\mathrm{J}$ Gastroenterol 13:2363-2368

Meplan C, Crosley LK, Nicol F et al (2007) Genetic polymorphisms in the human selenoprotein $\mathrm{P}$ gene determine the response of selenoprotein markers to selenium supplementation in a genderspecific manner (the SELGEN study). FASEB J 21:3063-3074

Meplan C, Crosley LK, Nicol F et al (2008) Functional effects of a common single-nucleotide polymorphism (GPX4c718t) in the glutathione peroxidase 4 gene: interaction with sex. Am J Clin Nutr 87:1019-1027

Meplan C, Nicol F, Burtle BT et al (2009) Relative abundance of selenoprotein $\mathrm{P}$ isoforms in human plasma depends on genotype, Se intake, and cancer status. Antioxid Redox Signal 11:26312640

Moriarty PM, Reddy CC, Maquat LE (1998) Selenium deficiency reduces the abundance of mRNA for Se-dependent glutathione peroxidase 1 by a UGA-dependent mechanism likely to be nonsense codon-mediated decay of cytoplasmic mRNA. Mol Cell Biol 18:2932-2939

NAS (2000) National academy of science dietary reference intakes for vitamin $\mathrm{C}$, vitamin $\mathrm{E}$, selenium and carotenoids. National Academy Press, Washington

Nogueira CW, Rocha JB (2011) Toxicology and pharmacology of selenium: emphasis on synthetic organoselenium compounds. Arch Toxicol. doi:10.1007/s00204-011-0720-3

Oldfield JE (1999) Selenium world atlas. Selenium-Tellurium Development Association, Brussels

Pagmantidis V, Meplan C, van Schothorst EM et al (2008) Supplementation of healthy volunteers with nutritionally relevant amounts of selenium increases the expression of lymphocyte protein biosynthesis genes. Am J Clin Nutr 87:181-189

Papp LV, Lu J, Holmgren A, Khanna KK (2007) From selenium to selenoproteins: synthesis, identity, and their role in human health. Antioxid Redox Signal 9:775-806

Ravn-Haren G, Bugel S, Krath BN et al (2008a) A short-term intervention trial with selenate, selenium-enriched yeast and selenium-enriched milk: effects on oxidative defence regulation. Br J Nutr 99:883-892

Ravn-Haren G, Krath BN, Overvad K et al (2008b) Effect of longterm selenium yeast intervention on activity and gene expression of antioxidant and xenobiotic metabolizing enzymes in healthy elderly volunteers from the Danish Prevention of Cancer by Intervention by Selenium (PRECISE) Pilot Study. Br J Nutr 99:1190-1198

Rayman MP (2004) The use of high-selenium yeast to raise selenium status: how does it measure up? Br J Nutr 92:557-573
Reeves MA, Hoffmann PR (2009) The human selenoproteome: recent insights into functions and regulation. Cell Mol Life Sci 66:2457-2478

Reszka E, Gromadzinska J, Jablonska E et al (2009) Level of selenoprotein transcripts in peripheral leukocytes of patients with bladder cancer and healthy individuals. Clin Chem Lab Med 47:1125-1132

Schomburg L, Schweizer U (2009) Hierarchical regulation of selenoprotein expression and sex-specific effects of selenium. Biochim Biophys Acta 1790:1453-1462

Schriever SC, Barnes KM, Evenson JK et al (2009) Selenium requirements are higher for glutathione peroxidase-1 mRNA than gpx1 activity in rat testis. Exp Biol Med 234:513-521

Schwarz K, Foltz CM (1958) Factor 3 activity of selenium compounds. J Biol Chem 233:245-251

Shchedrina VA, Zhang Y, Labunskyy VM et al (2010) Structurefunction relations, physiological roles, and evolution of mammalian ER-resident selenoproteins. Antioxid Redox Signal 12:839-849

Small-Howard AL, Berry MJ (2005) Unique features of selenocysteine incorporation function within the context of general eukaryotic translational processes. Biochem Soc Trans 33: 1493-1497

Squires JE, Stoytchev I, Forry EP et al (2007) SBP2 binding affinity is a major determinant in differential selenoprotein mRNA translation and sensitivity to nonsense-mediated decay. Mol Cell Biol 27:7848-7855

Sun W, Wang X, Zou X et al (2010) Selenoprotein P gene r25191 g/a polymorphism and quantification of selenoprotein $\mathrm{P}$ mRNA level in patients with Kashin-Beck disease. Br J Nutr 104:1283-1287

Sunde RA (2010) Molecular biomarker panels for assessment of selenium status in rats. Exp Biol Med 235:1046-1052

Sunde RA, Hadley KB (2010) Phospholipid hydroperoxide glutathione peroxidase $(\mathrm{Gpx} 4)$ is highly regulated in male turkey poults and can be used to determine dietary selenium requirements. Exp Biol Med 235:23-31

Sunde RA, Thompson KM (2009) Dietary selenium requirements based on tissue selenium concentration and glutathione peroxidase activities in old female rats. J Trace Elem Med Biol 23:132-137

Sunde RA, Paterson E, Evenson JK et al (2008) Longitudinal selenium status in healthy British adults: assessment using biochemical and molecular biomarkers. Br J Nutr 99(Suppl 3):S37-S47

Sunde RA, Raines AM, Barnes KM et al (2009) Selenium status highly regulates selenoprotein mRNA levels for only a subset of the selenoproteins in the selenoproteome. Biosci Rep 29:329-338

Thomson CD (2004) Assessment of requirements for selenium and adequacy of selenium status: a review. Eur J Clin Nutr 58:391-402

Vincent JL, Forceville X (2008) Critically elucidating the role of selenium. Curr Opin Anaesth 21:148-154

Visvikis-Siest S, Marteau J-B, Samara A et al (2007) Peripheral blood mononuclear cells (PBMCs): a possible model for studying cardiovascular biology systems. Clin Chem Lab Med 45:1154-1168

Waters DJ, Chiang EC, Cooley DM et al (2004) Making sense of sex and supplements: differences in the anticarcinogenic effects of selenium in men and women. Mutat Res 551:91-107

Weiss SL, Sunde RA (1998) Cis-acting elements are required for selenium regulation of glutathione peroxidase-1 mRNA levels. RNA 4:816-827

Xia Y, Hill KE, Byrne DW et al (2005) Effectiveness of selenium supplements in a low-selenium area of China. Am J Clin Nutr 81:829-834

Xia Y, Hill KE, Li P et al (2010) Optimization of selenoprotein P and other plasma selenium biomarkers for the assessment of the 
selenium nutritional requirement: a placebo-controlled, doubleblind study of selenomethionine supplementation in seleniumdeficient Chinese subjects. Am J Clin Nutr 9:525-531

Zachara BA, Gromadzinska J, Wasowicz W et al (2006) Red blood cell and plasma glutathione peroxidase activities and selenium concentration in patients with chronic kidney disease: a review. Acta Biochim Pol 53:663-677
Zhou J-C, Zhao H, Li J-G et al (2009) Selenoprotein gene expression in thyroid and pituitary of young pigs is not affected by dietary selenium deficiency or excess. J Nutr 139:1061-1066 